\title{
Measures to improve system stability of island microgrid
}

\author{
Deng Hua ${ }^{1, a}{ }^{*}$, Li Guanglei, ${ }^{2, b}$, Li Sun ${ }^{3, c}$, Wang Youjie ${ }^{4, d}$ and Hu Hengrui ${ }^{5, e}$ \\ ${ }^{1}$ State Grid Yantai Power Supply Company, Yantai, China \\ ${ }^{2}$ State Grid Shandong Electric Power Research Institute, Jinan, China \\ ${ }^{3}$ State Grid Shandong Electric Power Company, Jinan, China \\ ${ }^{4}$ Shandong Luneng Software Technology Co.,Ltd, Jinan, China \\ ${ }^{5}$ State Grid Weifang Power Supply Company, Weifang, China \\ a214672717@qq.com, b lovexjtulgl@126.com, c qianli917a@sina.com, ${ }^{\mathrm{d}}$ sdzhc@sohu.com, \\ ell@163.com
}

\begin{abstract}
Keywords: system operation; control strategy; functional design; system test.
Abstract. In order to ensure the security and stability operation of microgrid, this paper construct simulation models of renewable energy generation grid, propose the control strategy of the grid-connected and microgrid operation, and analyze these control strategy based on Power Systems Computer Aided Design. Results validate this feasibility of control strategies, which presents important references for operational control of island microgrid with renewable energy resources. Therefore, the study on island grid with renewable energy resources is very meaningful.
\end{abstract}

\section{Introduction}

With the rapid development of China's marine industry, marine resources development and utilization is listed as a major strategic initiative of economic construction. Island preservation, exploitation and management have been become the focus of the project in new century. Different from the traditional power system, island microgrid combine distributed power, load and energy storage device together [1][2][3]. This has become a new type of power exchange system, including electric energy collection, transmission of electrical energy, electrical energy storage and power distribution. There has not been an in - depth study of Modeling and Simulation of containing a variety of intermittent renewable energy island system in domestic research[4][5].

In order to ensure the security and stability operation of microgrid, this paper constructs simulation models of renewable energy generation grid. Be aimed at the different operating conditions of intermittent renewable energy island system, this paper propose the control strategy of the grid-connected and microgrid operation, and analyze these control strategy based on PSACD.

\section{Modeling analysis}

The power grid structure of renewable sources The structure of island grid is shown as below. This system contains $35 \mathrm{kV}, 10 \mathrm{kV}$ two voltage levels and connects the large power grid through $110 \mathrm{kV}$ submarine cables. Under the condition of synchronization, $110 \mathrm{kV}$ line is the only source of island. When the grid broke down, $110 \mathrm{kV}$ submarine cables will switch turn off. So this island power grid will form an isolated system.

Island microgrid with renewable energy resources usually includes a variety of distributed power supply, such as Wind power, photovoltaic, energy storage and diesel generator. Distributed power comprises a significant proportion of tenants in island microgrid. Power load is divided into conventional load and seawater desalination load. The typical structure of intermittent renewable energy grid is shown in Fig.1. 


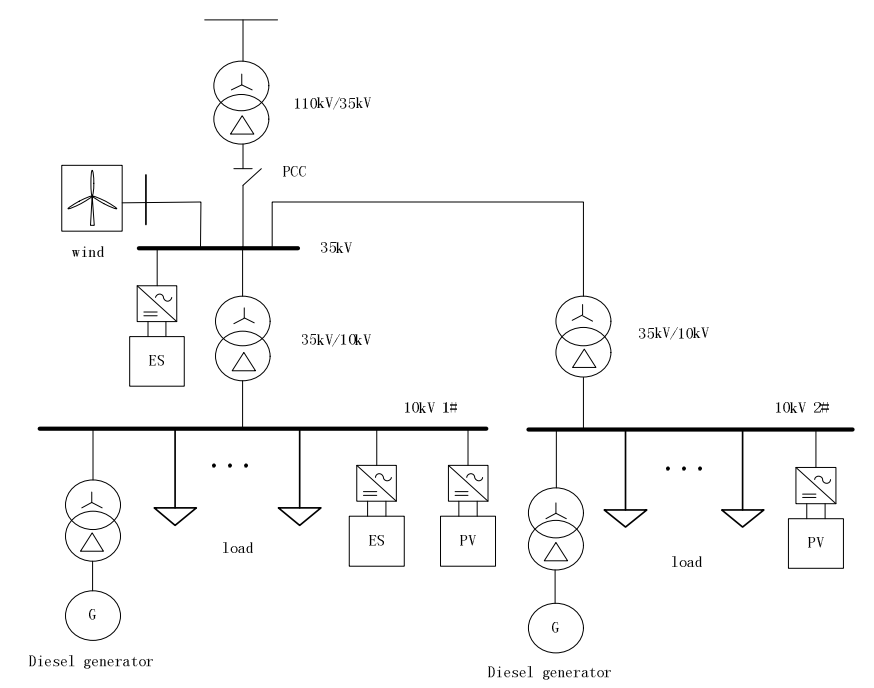

Fig.1 Power network structure of the renewable energy grid

Wind farm Wind DFIG has many advantages, such as adjust amplitude, phase and frequency of rotor excitation current, control real power and reactive power, have excellent network characteristics[6][7]. Target management of grid-side converter should stabilize the DC bus voltage and adjust the power factor. This control method and control block diagram is treading a similar path to machine side converter. While target management of machine side converter should output active power and reactive power according the instruction. Under normal circumstances, the power command is the largest wind power obtained tracking. While reactive power can be set a value on demand. This control graph of control system is shown in Fig.2.

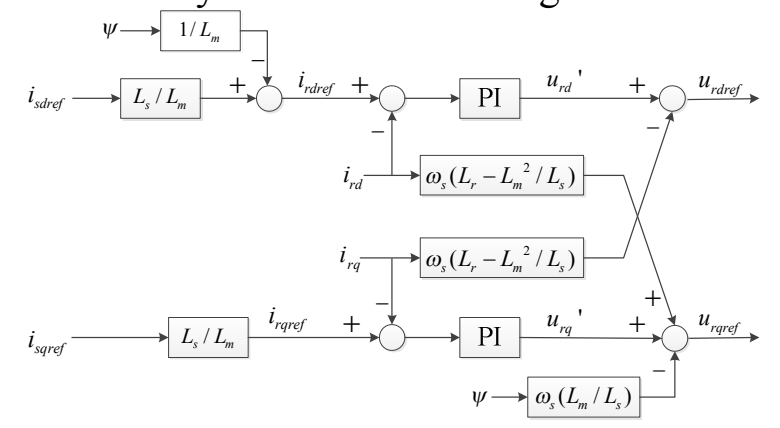

Fig.2 Control block diagram of machine side converter of wind turbines

Among them: Lr、Ls and Lm separately represent rotor inductance, the stator and rotor mutual inductance, Ird、Irq、Isd and Isq separately represent rotor current and stator current of DFIG in the dq coordinate system, represent magnetic flux, PI is control algorithm, Udrref and Uqref separately represent rotor current and stator current of machine side converter in the dq coordinate system.

Power station model of PV Equivalent circuit of photovoltaic cell[8][9] is shown in Fig.3.

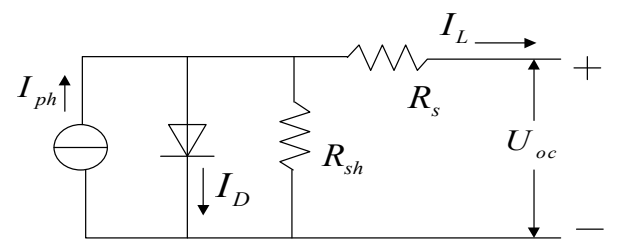

Fig.3 Equivalent circuit of photovoltaic cell

Among them: Iph、ID、Rsh、RS、IL and UOC separately represent photocurrent, parallel diode current flows, parallel equivalent resistance, equivalent series resistance, photocurrent and the PV array voltage[10].

$$
I_{L}=I_{p h}-I_{0}\left\{\exp \left[\frac{q\left(U_{o c}+I_{L} R_{s}\right)}{A k T}\right]-1\right\}-\frac{U_{D}}{R_{s h}}
$$




$$
\begin{gathered}
I_{0}=I_{o r}\left(\frac{T}{T_{r}}\right) \exp \left[\frac{q E_{G}}{B k}\left(\frac{1}{T_{r}}-\frac{1}{T}\right)\right] \\
I_{p h}=\left[I_{s c}+K_{t}(T-298)\right] \frac{G}{1000}
\end{gathered}
$$

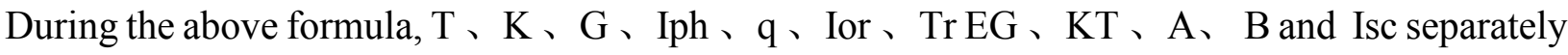
represent temperature, boltzmann constant, sunshine intensity, light green under current, electronic power, diode reverse saturation current, the reference temperature, the band gap of a semiconductor material, the short-circuit current temperature coefficient, the ideal factor and short-circuit current standard test conditions. Output characteristics of the photovoltaic array is affected by solar radiation, temperature and other factors, so reference voltage $\mathrm{Vdc}$ is acquired by the maximum power point tracking algorithm, in order to improve the power generation efficiency of the photovoltaic array.

\section{Islanding control schemes}

When undersea submarine cable fails, it takes longer timer to restore power compared to the mainland power grid. In this case, the system must control effectively energy storage system and diesel generator to ensure the normal operation of the island grid within eliminates fault time. When diesel generator is used as the main power supply system, islanding control schemes are shown as follows. When the island grid is in the islanding operation state grid return to island running, diesel generator immediately put into operation and carry significant load gradually restore the system voltage and frequency. At the same time, the energy storage unit work in droop controls to prevent intermittent power, which is restored electricity generation grid load island net negative occurrence.

\section{System Test}

When the $110 \mathrm{kV}$ line fails, island grid appear a brief power failure, quickly build system voltage by the energy storage unit. Meanwhile, the diesel generators are in hot standby state. At the time of $1 \mathrm{~s}$, island grid load suddenly increases. The energy storage unit is close to the upper limit of the output power $200 \mathrm{~kW}$, then diesel generator increase output in order to achieve no difference frequency and voltage regulation. Power variation and voltage is shown in Fig.4 and Fig.5.

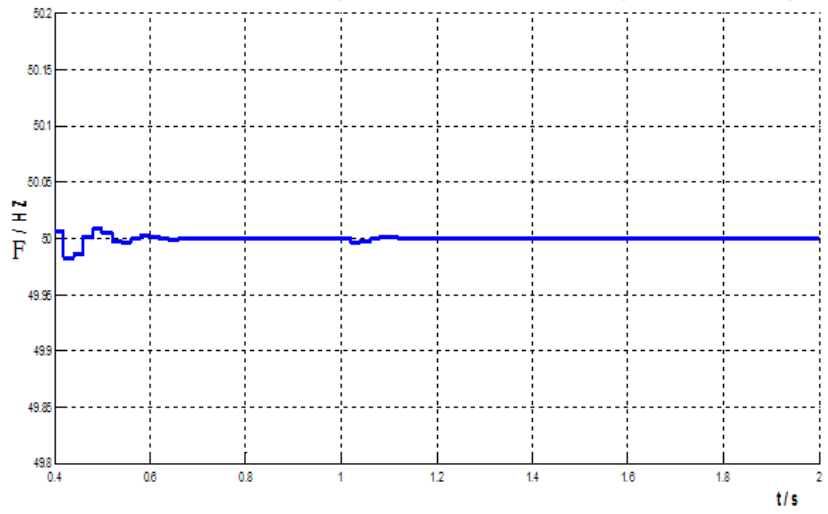

Fig.4 Power frequency

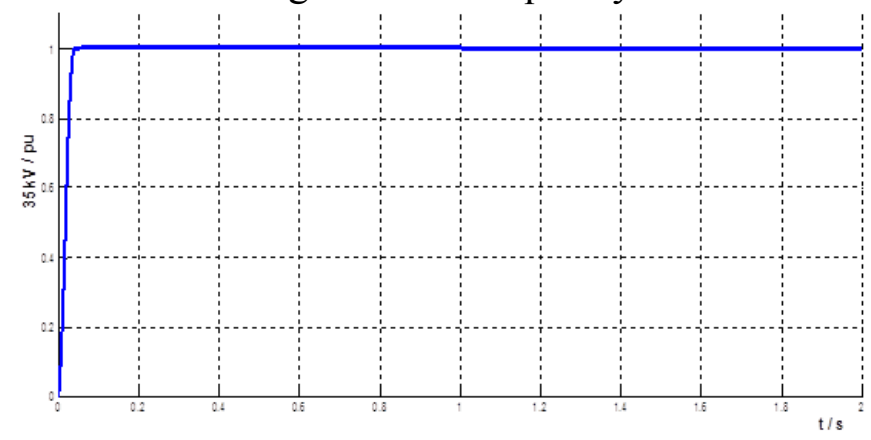

Fig. $535 \mathrm{kV}$ bus voltage 
From the above results, the energy storage unit and diesel generator can be coordinated control to ensure the system voltage and frequency stability and also reduces the risk of overloading the storage unit.

\section{Summary}

Different from the traditional power system, island microgrid combine distributed power, load and energy storage device together. When active intermittent power adjustment of the island grid exceeds the regulating capacity of battery storage systems, this system can increase or reduce the load of desalination to increase grid - connected wind capacity. Using PSCAD simulation platform, operation control strategies are justified under the conditions of the grid connection and island running. The results show strategies proposed can ensure the system voltage and frequency stability..

\section{References}

[1] Li Bihui, Shen Hong, Tong Yong, et al, "Impacts of energy storage capacity configuration of HPWS to active power characteristic and its relevant indices," Power System Technology, vol. 35, Dec. 2001, pp. 123-128.

[2] IEEE standard for interconnecting distributed resources with electric power systems. IEEE Std1547-2003 . 2003.

[3] Lu Zongxiang, Wang Caixia, Min Yong, et al, "Overviewon microgrid research.," Automation of Electric Power Systems, vol. 31,May. 2007, pp. 100-107.

[4] Zhang Mingrui, Du Zhichao, Li Na, et al, "Control strategies of frequency stability for islanding high-voltage microgrids., " Automation of Electric Power Systems, vol. 32,May. 2012, pp. 20-26.

[5] Li Fudong, Wu Min, "An improved control strategy of load distribution in an autonomous microgrid,” Proceedings of the CSEE, vol. 46,Jan. 2011, pp. 18-25.

[6] Wang Cheng-shan, Xiao Zhao-xia,Wang Shou-xiang, "Syn-thetical control and analysis of microgrid," Automation of Electric Power Systems, vol. 32, Dec. 2008, pp. 98-103.

[7] Wang He, Li Guoqing, "Conrol strategy of microgrid with different DG types," Electric Power Automation Equipment, vol. 32,Feb. 2012, pp. 19-23.

[8] Guo Y, Fang Y, "Electricity cost saving strategy in data centers by using energy storage," IEEE Transactions on Parallel and Distributed Systems, vol. 24,May. 2013, pp. 1149-1160.

[9] Amjady N, Keynia F, Zareipour H, "Short-term load forecast of microgrids by a new bilevel prediction strategy," IEEE Transactions on Smart Grid, vol.1,Jan. 2010, pp. 286-294.

[10] Varaiya P P, Wu F F, Bialek J W, “Smart Operation of Smart Grid: Risk -Limiting Dispatch,” Proceedings of the IEEE, vol. 99, Dec. 2011, pp. 40-57. 